\title{
A Note on the Dependence Conditions for Stationary Normal Sequences
}

\author{
Hyemi Choi ${ }^{1, a}$ \\ ${ }^{a}$ Department of Statistics, Chonbuk National University, Korea
}

\begin{abstract}
Extreme value theory concerns the distributional properties of the maximum of a random sample; subsequently, it has been significantly extended to stationary random sequences satisfying weak dependence restrictions. We focus on distributional mixing condition $D\left(u_{n}\right)$ and the Berman condition based on covariance among weak dependence restrictions. The former is assumed for general stationary sequences and the latter for stationary normal processes; however, both imply the same distributional limit of the maximum of the normal process. In this paper $D\left(u_{n}\right)$ condition is shown weaker than Berman's covariance condition. Examples are given where the Berman condition is satisfied but the distributional mixing is not.
\end{abstract}

Keywords: Berman condition, covariance, extreme value theory, mixing condition, stationary normal sequence.

\section{Introduction}

Classical extreme value theory concerns the distributional properties of the maximum of independent and identically distributed (iid) random variables. Its centerpiece is the "extremal types theorem" (ETT). It asserts that in the iid setting the only three possible types of non-degenerate limiting distributions $G$ for the maximum are

$$
\begin{aligned}
& \text { I: } \quad G(x)=\exp \left(-e^{-x}\right), \quad-\infty<x<\infty \text {; } \\
& \text { II: } G(x)=\exp \left(-x^{-\alpha}\right), \quad 0<x<\infty, \alpha>0 \text {; } \\
& \text { III: } G(x)=\exp \left(-(-x)^{\alpha}\right), \quad-\infty<x<0, \quad \alpha>0 \text {, }
\end{aligned}
$$

where $x$ may be replaced by $a x+b$ for real $a>0, b \in \mathbb{R}$. The family labeled I is also often called as the double exponential or Gumbel family. Types II and III are widely known as the Fréchet and Weibull families.

The theory has been significantly extended to include dependence. In particular, the ETT still holds for a stationary process satisfying weak dependence restrictions. Leadbetter et al. (1983) provides proof of an ETT under a distributional mixing condition $D\left(u_{n}\right)$. We focus on the condition $D\left(u_{n}\right)$ in this paper and the definition of the $D\left(u_{n}\right)$ condition will be given in Section 2 . Leadbetter and Rootzén (1998), Leadbetter et al. (2000) and Turkman (2006) obtained the extremal results for random fields satisfying the extended $D\left(u_{n}\right)$-condition to random fields. These conditions can be written in terms of finite dimensional distribution functions.

\footnotetext{
${ }^{1}$ Department of Statistics (Institute of Applied Statistics), Chonbuk National University, Jeonbuk 54896, Korea.

E-mail: hchoi@jbnu.ac.kr
}

Published 30 November 2015 / journal homepage: http://csam.or.kr

(c) 2015 The Korean Statistical Society, and Korean International Statistical Society. All rights reserved. 
We consider a stationary normal sequence $\left\{\xi_{i}\right\}$ with mean zero and variance one in this paper. Let $M_{n}=\max \left(\xi_{1}, \xi_{2}, \ldots, \xi_{n}\right)$. It is well-known that in the iid setting $M_{n}$ has a limiting distribution of the double exponential type among three extremal types,

$$
P\left\{a_{n}\left(M_{n}-b_{n}\right) \leq x\right\} \rightarrow \exp \left(-e^{-x}\right) \quad \text { as } n \rightarrow \infty
$$

with $a_{n}=(2 \log n)^{1 / 2}$ and $b_{n}=a_{n}-\left(2 a_{n}\right)^{-1}\{\log \log n+\log 4 \pi\}$. This convergence also holds for stationary normal case satisfying some dependence conditions. The dependence condition of normal sequences is often represented by covariance. Berman (1964) gave simple conditions on covariances of $\left\{\xi_{i}\right\}$ to ensure (1.1). One of Berman's such conditions is that

$$
\rho_{n} \log n \rightarrow 0 \text { as } n \rightarrow \infty,
$$

where $\rho_{n}=\mathrm{E}\left(\xi_{i} \xi_{i+n}\right)$ is the covariance of the normal process $\left\{\xi_{i}\right\}$. It has been considered as a fundamental sufficient condition in proving distributional results for extreme values of correlated data, see Leadbetter et al. (1983), Lindgren and Rootzén (1987), Embrechts et al. (1999) and recently Turner and Chareka (2012). The convergence of maxima to the double exponential distribution is also obtained in the case of stationary normal sequences satisfying the $D\left(u_{n}\right)$ condition. It is shown in Leadbetter et al. (1983) that the Berman condition (1.2) implies the condition $D\left(u_{n}\right)$ for normal sequences.

Section 2 provides the $D\left(u_{n}\right)$ condition and a sketch of proof that the Berman condition (1.2) is no weaker than the condition $D\left(u_{n}\right)$. In Section 3, some examples satisfying the Berman but not the $D\left(u_{n}\right)$ condition are given. Consequently, the $D\left(u_{n}\right)$ mixing condition is shown to be strictly weaker than Berman's condition (1.2) for the convergence of maxima of normal sequences to the double exponential distribution.

\section{Mixing Conditions}

The dependence restriction for stationary sequences is often given by using "mixing function". We focus on the condition $D\left(u_{n}\right)$ here. The condition $D\left(u_{n}\right)$ is a weakened version of such mixing conditions for extreme value theory. It considers only the events of the form $\left\{\xi_{i} \leq u_{n}\right\}$ or their intersections for a certain sequence of values $\left\{u_{n}\right\}$. For brevity, we write $F_{i_{1}, \ldots, i_{k}}(u)$ for $F_{i_{1}, \ldots, i_{k}}(u, \ldots, u)$ if $F_{i_{1}, \ldots, i_{k}}\left(x_{1}, \ldots, x_{k}\right)$ denotes the joint distribution function of random variables $\xi_{i_{1}}, \ldots, \xi_{i_{k}}$. For sequences of constants $\left\{a_{n}\right\}$ and $\left\{b_{n}\right\}$ we write $a_{n}=o\left(b_{n}\right)$ if $a_{n} / b_{n} \rightarrow 0$ and $a_{n}=O\left(b_{n}\right)$ if $a_{n} / b_{n}$ is bounded as $n \rightarrow \infty$. Leadbetter et al. (1983) defines $D\left(u_{n}\right)$ condition as: The condition $D\left(u_{n}\right)$ will be said to hold if

$$
D\left(u_{n}\right)=\sup \left|F_{i_{1}, \ldots, i_{p}, j_{1}, \ldots, j_{p^{\prime}}}\left(u_{n}\right)-F_{i_{1}, \ldots, i_{p}}\left(u_{n}\right) F_{j_{1}, \ldots, j_{p^{\prime}}}\left(u_{n}\right)\right|=o(1)
$$

where the supremum is taken over any integers $1 \leq i_{1}<\cdots<i_{p}<j_{1}<\cdots<j_{p^{\prime}}$ satisfying $j_{1}-i_{p} \geq l_{n}=o(n)$ and $l_{n} \rightarrow \infty$ as $n \rightarrow \infty$.

On the other hand, the dependence restrictions for stationary normal sequences are often represented by covariances. Among them is the Berman condition (1.2) known as a "very weak" sufficient condition for the convergence of maxima of normal sequences to double exponential distribution. Let $\Phi$ denote the standard normal distribution function and $\left\{u_{n}\right\}$ a sequence of constants such that $n\left(1-\Phi\left(u_{n}\right)\right)$ converges to a finite limit. By using (3.2), it can be shown that the Berman condition (1.2) implies that

$$
n \sum_{j=1}^{n}\left|\rho_{j}\right| \exp \left(-\frac{u_{n}^{2}}{1+\left|\rho_{j}\right|}\right)=o(1)
$$


Lemma 4.3.2 of Leadbetter et al. (1983) for the detail proof. By application of Normal Comparison Lemma we also have that for some constant $K$

$$
\left|F_{i_{1}, \ldots, i_{s}}\left(u_{n}\right)-\Phi^{s}\left(u_{n}\right)\right| \leq K n \sum_{j=1}^{n}\left|\rho_{j}\right| \exp \left(-\frac{u_{n}^{2}}{1+\left|\rho_{j}\right|}\right)
$$

and hence

$$
D\left(u_{n}\right) \leq 3 K n \sum_{j=1}^{n}\left|\rho_{j}\right| \exp \left(-\frac{u_{n}^{2}}{1+\left|\rho_{j}\right|}\right)
$$

from the definition of $D\left(u_{n}\right)$. Thus by (2.2) and (2.3) we can see that the Berman condition implies the condition $D\left(u_{n}\right)$. The distribution of the maximum of stationary normal sequences converges to the double exponential distribution under $D\left(u_{n}\right)$ as under the Berman condition; in addition, the former condition is actually weaker than the latter since it is possible to give examples where the latter holds but the former does not as will be seen in Section 3.

\section{Examples}

To construct such examples, we define sequences of standard normal random variables with covariances $\left\{\rho_{n, v}, n=0,1,2, \ldots\right\}$ for each $v=3,4, \ldots$. The following examples of covariance $\rho_{n, v}$ were originally suggested for continuous normal processes by Mittal (1979) but are applied to normal sequences here. The remainder of this section gives the verification of these examples. The covariances $\rho_{n, v}$ will be constructed by multiplication of two different covariance sequences $\gamma_{n}$ and $\delta_{n}$ defined in the following. It will be shown that for each $v=3,4, \ldots,\left\{\rho_{n, v}\right\}$ satisfies the $D\left(u_{n}\right)$ condition but not the Berman.

First, we consider

$$
\gamma(t)= \begin{cases}\sqrt{1-\frac{|t|}{2 e^{2}}}, & |t| \leq e^{2}, \\ \frac{1}{\sqrt{\log |t|}}, & |t|>e^{2} .\end{cases}
$$

This is easily seen to satisfy Pólya's criteria, i.e. $\gamma(t)$ is real, nonnegative, continuous and convex on $[0, \infty)$ and satisfies $\gamma(t)=\gamma(-t)$ and $\gamma(0)=1$. Hence $\gamma(t)$ is a covariance function. Next, we consider the sum of countably many independently distributed random variables which take only two values $-a^{j}, a^{j}$ with probability $1 / 2$ respectively. Its characteristic function is found as

$$
\delta_{a}(t)=\prod_{j=1}^{\infty}\left(\frac{e^{i a^{j} t}+e^{-i a^{j} t}}{2}\right)=\prod_{j=1}^{\infty} \cos \left(a^{j} t\right) .
$$

The $\delta_{a}(t)$ defines a (singular) covariance function for $0<a<1 / 2$ (Lukcas, 1970). Since the product of two covariance functions is again a valid covariance function, the multiplication of $\gamma(t)$ and $\delta_{a}(t)$ can define a covariance function for $0<a<1 / 2$. Now, we have the covariance $\left\{\rho_{n, v}, n=0,1,2, \ldots\right\}$ 
for each $v=3,4, \ldots$ defined as:

$$
\begin{aligned}
\rho_{n, v}= & \gamma(n) \delta_{\frac{1}{v}}(n) \\
= & \begin{array}{ll}
\left(1-\frac{n}{2 e^{2}}\right)^{\frac{1}{2}} \prod_{j=1}^{\infty} \cos \left(v^{-j} t\right), & n \leq e^{2}, \\
(\log n)^{-\frac{1}{2}} \prod_{j=1}^{\infty} \cos \left(v^{-j} t\right), & n>e^{2} .
\end{array}
\end{aligned}
$$

We note that since $\lim \sup _{n \rightarrow \infty}\left|\delta_{1 / v}(n)\right|>0$ (Wintner, 2013),

$$
\limsup _{n \rightarrow \infty} \rho_{n, v} \log n=\limsup _{n \rightarrow \infty}(\log n)^{\frac{1}{2}} \delta_{\frac{1}{v}}(n)=\infty .
$$

Thus we see that Berman's condition does not hold for $\left\{\rho_{n, v}\right\}$.

However, the $D\left(u_{n}\right)$ condition does hold for $\left\{\rho_{n, v}\right\}$, which will be shown by proving

$$
n \sum_{j=1}^{n}\left|\rho_{j, v}\right| \exp \left(-\frac{u_{n}^{2}}{1+\left|\rho_{j, v}\right|}\right)=o(1)
$$

since by $(2.3)$

$$
D\left(u_{n}\right) \leq 3 K n \sum_{j=1}^{n}\left|\rho_{j, v}\right| \exp \left(-\frac{u_{n}^{2}}{1+\left|\rho_{j, v}\right|}\right) .
$$

First, we note that the fact that if the covariance $\left|\rho_{n}\right| \rightarrow 0$ as $n \rightarrow \infty$, then $\left|\rho_{n}\right| \neq 1$ for any $n \neq 0$ and hence it is bounded away from 1 for all $n>0$.

Let $\tau=\sup _{j \geq n}\left|\rho_{j, v}\right|$, which is clearly less than 1 since $\rho_{n, v} \rightarrow 0$ as $n \rightarrow \infty$ as noted above. Define $\alpha$ as a constant such that $0<\alpha<(1-\tau) /(1+\tau)$. For notational simplicity, we use $K$ as a constant whose value may change from line to line. Since $n\left(1-\Phi\left(u_{n}\right)\right)$ converges to a finite limit, we can use the fact that for sufficiently large $n$

$$
\exp \left(-\frac{u_{n}^{2}}{2}\right)=\frac{K u_{n}}{n}(1+o(1)) \quad \text { and } \quad u_{n}=\sqrt{2 \log n}(1+o(1)) .
$$

We split the sum in (3.1) into two parts, the first for $1 \leq j \leq\left[n^{\alpha}\right]$ and the second for $\left[n^{\alpha}\right]<j \leq n$. By applying (3.2) we see that the first sum is dominated by

$$
\begin{aligned}
n n^{\alpha} \exp \left(-\frac{u_{n}^{2}}{1+\tau}\right) & =n^{1+\alpha} \exp \left(-\frac{u_{n}^{2}}{2}\right)^{\frac{2}{1+\tau}} \leq K n^{1+\alpha}\left(\frac{u_{n}}{n}\right)^{\frac{2}{1+\tau}} \\
& \leq K n^{1+\alpha-\frac{2}{1+\tau}}(\log n)^{\frac{1}{1+\tau}},
\end{aligned}
$$

which tends to zero from the choice of $\alpha$ and hence so does the first part of the sum.

To deal with the second part let $A_{n 1}=\left\{\left[n^{\alpha}\right]<j \leq n ;\left|\rho_{j, v}\right|>(\ln j)^{-2}\right\}$ and $A_{n 2}=\left\{\left[n^{\alpha}\right]<j \leq\right.$ $\left.n ;\left|\rho_{j, v}\right| \leq(\ln j)^{-2}\right\}$, where $[x]$ denotes the greatest integer less than or equal to $x$. Now, we have for the second part of the sum in (3.1)

$$
\begin{aligned}
n \sum_{j=\left[n^{\alpha}\right]+1}^{n}\left|\rho_{j, v}\right| \exp \left(-\frac{u_{n}^{2}}{1+\left|\rho_{j, v}\right|}\right) & =n \sum_{m \in A_{n 1}}\left|\rho_{j, v}\right| \exp \left(-\frac{u_{n}^{2}}{1+\left|\rho_{j, v}\right|}\right)+n \sum_{m \in A_{n 2}}\left|\rho_{j, v}\right| \exp \left(-\frac{u_{n}^{2}}{1+\left|\rho_{j, v}\right|}\right) \\
& =S_{1}+S_{2}, \text { say. }
\end{aligned}
$$


Using the same reasoning as for the first part of the sum in (3.1) we have

$$
S_{1} \leq K\left|A_{n 1}\right| n \exp \left\{-\frac{u_{n}^{2}}{1+\tau\left(n^{\alpha}\right)}\right\} \leq K\left|A_{n 1}\right| n^{1-\frac{2}{\left(1+\tau\left(n^{\alpha}\right)\right)}}(\log n)^{\frac{1}{\left(1+\tau\left(n^{\alpha}\right)\right)}},
$$

where $\tau(l)=\sup _{j \geq l}\left|\rho_{j, v}\right|$. We claim that $\left|A_{n 1}\right|=o\left(n^{\kappa}\right)$ with $\kappa=1-(\log 2 / 2 \log v)$. If not, $\left|\rho_{j, v}\right|>$ $(\log j)^{-2}$ on a set of size $O\left(n^{\kappa}\right)$, which implies $\sum_{j=0}^{n}\left|\rho_{j, v}\right|^{2} \geq K n^{\kappa} /(\log n)^{4}$ contradicting $\sum_{j=0}^{n}\left|\rho_{j, v}\right|^{2} \leq$ $K n^{1-(\log 2 / \log v)}$. This inequality follows from the definition of $\left\{\rho_{n, v}\right\}$ and (4.2) in Appendix. Moreover, $\tau\left(n^{\alpha}\right)=o(1)$ because $\rho_{n, v}=o(1)$. Hence the rightmost-hand side of (3.3) tends to zero as $n \rightarrow \infty$.

Next, for $S_{2}$ we note that $\left|\rho_{j, v}\right| \leq(\log j)^{-2}$ for $j \in A_{n 2}$ and $(\log n)^{-2}$ is decreasing in $n \cdot \sup _{j \in A_{n 2}}\left|\rho_{j, v}\right| \leq$ $(\alpha \log n)^{-2}$ and hence by using (3.2) we have for sufficiently large $n$

$$
S_{2} \leq n^{2}(\alpha \log n)^{-2} \exp \left(-u_{n}^{2}\right) \exp \left\{u_{n}^{2}(\alpha \log n)^{-2}\right\}=K u_{n}^{2}(\alpha \log n)^{-2} \exp \left\{u_{n}^{2}(\alpha \log n)^{-2}\right\},
$$

which tends to zero. Thus (3.1) holds for $\left\{\rho_{n, v}\right\}$ and consequently the $D\left(u_{n}\right)$ condition holds by (2.3).

\section{Concluding Remarks}

As noted in Section 1, $M_{n}$ converges to the double exponential distribution for the stationary (standard) normal sequence under Berman condition or $D\left(u_{n}\right)$ condition;

$$
P\left\{a_{n}\left(M_{n}-b_{n}\right) \leq x\right\}=P\left\{M_{n} \leq \frac{x}{a_{n}}+b_{n}\right\} \rightarrow \exp \left(-e^{-x}\right) \quad \text { as } n \rightarrow \infty
$$

with $a_{n}=(2 \log n)^{1 / 2}$ and $b_{n}=a_{n}-\left(2 a_{n}\right)^{-1}\{\log \log n+\log 4 \pi\}$. In the $D\left(u_{n}\right)$, the sequence $\left\{u_{n}\right\}$ is necessary to satisfy that $n\left(1-\Phi\left(u_{n}\right)\right)$ converges to a finite limit. If we take $n\left(1-\Phi\left(u_{n}\right)\right)=e^{-x}$ and use the well-known relation for the tail of $\Phi$

$$
1-\Phi(u) \sim \frac{\phi(u)}{u} \quad \text { as } u \rightarrow \infty,
$$

where $\phi(u)$ denotes the standard normal probability density function, then after some computations we have

$$
\begin{aligned}
u_{n} & =(2 \log n)^{\frac{1}{2}}\left\{1+\frac{x-\frac{1}{2} \log 4 \pi-\frac{1}{2} \log \log n}{2 \log n}+o\left(\frac{1}{\log n}\right)\right\} \\
& =\frac{x}{a_{n}}+b_{n}+o\left(a_{n}^{-1}\right)
\end{aligned}
$$

Hence (4.1) would be written as $P\left\{M_{n} \leq u_{n}\right\} \rightarrow \exp \left(e^{-x}\right)$ for such $u_{n}$ under Berman or $D\left(u_{n}\right)$ condition.

We also remark that in general stationary case, the same limiting distribution of $M_{n}$ as in the iid would not be assured only under the $D\left(u_{n}\right)$ condition but the "local" condition is additionally required for it. In stationary normal case, the finite convergence of $n\left(1-\Phi\left(u_{n}\right)\right)$ implies the "local" condition.

\section{Appendix}

We claim for $v=3,4, \ldots$

$$
\sum_{j=0}^{n}\left|\delta_{\frac{1}{v}}(j)\right|^{2}=O\left(n^{1-\left(\frac{\log 2}{\log v}\right)}\right)
$$


Proof: Since $\cos ^{2} x \leq 1$ and $\cos x=\left(e^{i x}+e^{-i x}\right) / 2$, we have for any integer $k$ and $j \neq 0$

$$
\left|\delta_{\frac{1}{v}}(j)\right|^{2}=\prod_{l=1}^{\infty}\left|\cos \left(j v^{-l}\right)\right|^{2}<\prod_{l=1}^{k}\left|\cos \left(j v^{-l}\right)\right|^{2}=\frac{C_{j}}{2^{2 k}},
$$

where $C_{j}=\prod_{l=1}^{k}\left|e^{i v^{-l} j}+e^{-i v^{-l} j}\right|^{2}$. Thus

$$
\sum_{j=0}^{n-1}\left|\delta_{\frac{1}{v}}(j)\right|^{2}<2^{-2 k} \sum_{j=0}^{n-1} C_{j}
$$

Now, we consider the upper bound for $C_{j}$. Let $I=\left\{\boldsymbol{i}=\left(i_{1}, \ldots, i_{k}\right) ; i_{l} \in\{-1,1\}, l=1, \ldots, k\right\}$. Clearly $|I|=2^{k}$. Let $t(\boldsymbol{i})=\sum_{l=1}^{k} i_{l} v^{-l}$ for $\boldsymbol{i}=\left(i_{1}, \ldots, i_{k}\right) \in I$ and let $I^{2}$ denote the set $\{(\boldsymbol{a}, \boldsymbol{b}) \in I \times I ; \boldsymbol{a} \neq \boldsymbol{b}\}$. Then

$$
C_{j}=\left|\sum_{\boldsymbol{a} \in I} \exp (i j t(\boldsymbol{a}))\right|^{2}=\sum_{\boldsymbol{a} \in I} 1+\sum_{(\boldsymbol{a}, \boldsymbol{b}) \in I^{2}} \cos (j(t(\boldsymbol{a})-t(\boldsymbol{b}))) .
$$

Also

$$
\begin{aligned}
\sum_{j=0}^{n-1} \sum_{(\boldsymbol{a}, \boldsymbol{b}) \in I^{2}} \cos (j(t(\boldsymbol{a})-t(\boldsymbol{b}))) & =\sum_{(\boldsymbol{a}, \boldsymbol{b}) \in I^{2}} \sum_{j=0}^{n-1} \cos (j(t(\boldsymbol{a})-t(\boldsymbol{b}))) \\
& =\sum_{(\boldsymbol{a}, \boldsymbol{b}) \in I^{2}} \cos \left(\frac{(n-1)(t(\boldsymbol{a})-t(\boldsymbol{b}))}{2}\right) \frac{\sin (n(t(\boldsymbol{a})-t(\boldsymbol{b})) / 2)}{\sin ((t(\boldsymbol{a})-t(\boldsymbol{b})) / 2)} \\
& <\sum_{(\boldsymbol{a}, \boldsymbol{b}) \in I^{2}} \frac{1}{|\sin ((t(\boldsymbol{a})-t(\boldsymbol{b})) / 2)|}
\end{aligned}
$$

which does not exceed $\pi \sum_{(\boldsymbol{a}, \boldsymbol{b}) \in I^{2}}|t(\boldsymbol{a})-t(\boldsymbol{b})|^{-1}$ by the inequalities that $\sin |x|>2|x| / \pi$ for $0<|x|<1$ and $|t(\boldsymbol{a})-t(\boldsymbol{b})| \leq \sum_{j=1}^{k}\left|a_{j}-b_{j}\right| v^{-j}<2$ for any $v=3,4, \ldots$ Hence we have

$$
\sum_{j=0}^{n-1} C_{j}<n 2^{k}+\sum_{(\boldsymbol{a}, \boldsymbol{b}) \in I^{2}} \frac{\pi}{|t(\boldsymbol{a})-t(\boldsymbol{b})|}
$$

Now, define $d(\boldsymbol{a}, \boldsymbol{b})=\inf \left\{j ; a_{j} \neq b_{j}\right\}$ for any $(\boldsymbol{a}, \boldsymbol{b}) \in I^{2}$ and $I_{j}^{2}=\left\{(\boldsymbol{a}, \boldsymbol{b}) \in I^{2} ; d(\boldsymbol{a}, \boldsymbol{b})=j\right\}$. By definitions, $1 \leq d(\boldsymbol{a}, \boldsymbol{b}) \leq k$ and $\left|I_{j}^{2}\right|=2^{2 k-j}, j=1, \ldots, k$. If $(\boldsymbol{a}, \boldsymbol{b}) \in I_{j}^{2}$ for some $j$, then

$$
|t(\boldsymbol{a})-t(\boldsymbol{b})|>\left|a_{j}-b_{j}\right| v^{-n}-2\left|v^{-j-1}+v^{-j-2}+\cdots+v^{-k}\right|>2 C v^{-j},
$$

where $C=(v-2) /(v-1)<1(C$ is positive and depends only on $v)$, so that

$$
\begin{aligned}
\sum_{(\boldsymbol{a}, \boldsymbol{b}) \in I^{2}} \frac{1}{|t(\boldsymbol{a})-t(\boldsymbol{b})|} & =\sum_{j=1}^{k} \sum_{(\boldsymbol{a}, \boldsymbol{b}) \in I_{j}^{2}} \frac{1}{|t(\boldsymbol{a})-t(\boldsymbol{b})|} \\
& <\sum_{j=1}^{k} \sum_{(\boldsymbol{a}, \boldsymbol{b}) \in I_{j}^{2}} \frac{v^{j}}{2 C}<=\sum_{j=1}^{k} \frac{v^{j}}{2 C}\left|I_{j}^{2}\right|=\frac{2^{2 k}}{2 C} \sum_{j=1}^{k}\left(\frac{v}{2}\right)^{j} .
\end{aligned}
$$


Thus it follows from (4.4) that

$$
\sum_{j=0}^{n-1} C_{j}<K 2^{k}\left(n+2^{k} \sum_{j=0}^{k}\left(\frac{v}{2}\right)^{j}\right)
$$

for some constant $K$ depending only on $v$. Hence from (4.3) we obtain that since $v=3,4, \ldots$

$$
\sum_{j=0}^{n-1}\left|\delta_{\frac{1}{v}}(j)\right|^{2}<\frac{C}{2^{k}}\left(n+v^{k}\right)
$$

Now, take $k=[\log n / \log v]$. Then since $2^{\log n / \log v}=n^{\log 2 / \log v}$ and $v^{\log n / \log v}=n$, we have that

$$
\sum_{j=0}^{n-1}\left|\delta_{\frac{1}{v}}(j)\right|^{2}=O\left(n^{1-\frac{\log 2}{\log v}}\right) .
$$

\section{References}

Berman, S. M. (1964). Limit theorems for the maximum term in stationary sequences, The Annals of Mathematical Statistics, 35, 319-329.

Embrecht, P., Klüppelberg, C. and Mikosch, T. (1999). Modelling Extremal Events for Insurance and Finance, Springer, New York.

Leadbetter, M. R., Lindgren, G. and Rootzén, H. (1983). Extremes and Related Properties of Random Sequences and Processes, Springer, New York.

Leadbetter, M. R. and Rootzén, H. (1998). On extreme value theory in stationary random fields, In Stochastic Processes and Related Topics, in Memory of Stamatis Cambanis, Eds. Karatzas et al., 275-285 Springer, Boston.

Leadbetter, M. R., Rootzén, H. and Choi, H. (2000). On central limit theory for random additive functions under weak dependence restrictions, In State of the Art in Probability and Statistics, de Gunst et al., eds. IMS Lecture Note-Monograph Series \#36, 464-476.

Lindgren, G. and Rootzén, H. (1987). Extreme values: theory and technical applications, Scandinavian Journal of Statistics, 14, 241-279.

Lukcas, E. (1970). Characteristic Functions, Hafner, New York.

Mittal, Y. (1979). A mixing condition for stationary Gaussian processes, The Annals of Probability, 7, 724-732.

Turkman, K. F. (2006). A note on the extremal index for space-time processes, Journal of Applied Probability, 43, 114-126.

Turner, R. and Chareka, P. (2012). A note on the Berman condition, The Brazilian Journal of Probability and Statistics, 25, 82-87.

Wintner, A. (2013). The Fourier Transforms of Probability Distributions, Literary Licensing, LLC. 
